\title{
Gastric Small Cell Carcinoma That Showed Complete Response to Irinotecan+Cisplatin Chemotherapy Treatment -A Case Report-
}

\author{
Yuichiro Tsukada $^{1)}$, Katsunori Tauchi ${ }^{1)}$, Masato Nakamura $^{1)}$, Kotaro Sasahara ${ }^{1)}$, Hirofumi Kishimoto ${ }^{1)}$, \\ Kayoko Higuchi ${ }^{2)}$ \\ ${ }^{1)}$ Department of Surgery, Aizawa Hospital, ${ }^{2)}$ Department of Pathology, Aizawa Hospital
}

\begin{abstract}
Generally, clinical outcome of gastric small cell carcinoma is extremely poor. In this paper, we report a case of a patient with gastric small cell carcinoma who completely responded to chemotherapy and showed a long survival without recurrence. A 56-year-old man was admitted to our hospital with symptoms of weight loss and a large mass in the upper abdomen. Esophagogastroduodenoscopy revealed a large tumor located in the posterior wall of the antrum of the stomach. Computed tomography revealed a large mass involving the stomach with regional lymph node metastasis and direct pancreatic invasion. Pathological and immunohistochemical analyses indicated a diagnosis of primary gastric small cell carcinoma. Curative resection was not considered likely, and hence, a combination chemotherapy consisting of irinotecan and cisplatin was administered. Following 4 cycles of chemotherapy, complete remission was confirmed. The treatment was stopped after 9 cycles as per the patient's request. The patient survived for more than 8 year without recurrence since the first diagnosis of cancer. This case suggests that combination chemotherapy with irinotecan and cisplatin, which is recognized as one of the standard regimens for small cell carcinoma of the lung, can provide clinical benefits for patients with gastric small cell carcinoma.
\end{abstract}

Key Words: gastric small cell carcinoma, irinotecan, cisplatin

(Received November 30, 2011; Accepted December 12, 2011)

\section{Introduction}

Gastric small cell carcinoma (GSCC) is a rare carcinoma of the stomach, accounting for only about $0.1 \%$ of all gastric cancers ${ }^{1)}$. The clinical outcome has been reported as extremely poor, and there is no standardized treatment based on evidence of efficacy ${ }^{2)}$.

Small cell carcinoma of the stomach is similar to small cell lung cancer in terms of its clinicopathological features ${ }^{3)}$. In case of small cell lung cancer, chemotherapy is considered an effective treatment strategy, and combination chemotherapy with irinotecan and cisplatin (IP) is a common treatment option ${ }^{4)}$.

In this report, we present a case of small cell carcinoma of the stomach that completely responded to IP.

\section{Case Report}

A 56-year -old man was admitted to our hospital with symptoms of weight loss of $20 \mathrm{~kg}$ over a 3-month period and a mass in the upper abdomen. He had nei-

Corresponding Author: Masato Nakamura, Department of Surgery, Aizawa Hospital, 2-5-1 Honjo, Matsumoto, Nagano 390-8510, Japan. TEL: +81-263-338600, Fax: +81-263-32-6763, E-mail: geka-dr7@ai-hosp.or.jp ther a relevant past history nor a contributory family history. Physical examination revealed a large mass in the upper abdomen and tenderness. Laboratory findings after admission revealed mild anemia and elevation of neuron-specific enolase (NSE) and immunosuppressive acidic protein (110 ng/mL and $793 \mu \mathrm{g} / \mathrm{mL}$, respectively). Carcinoembryonic antigen and cancer antigen 19-9 were not elevated. Esophagogastroduodenoscopy (EGD) showed a large ulcerated tumor in the posterior wall of the antrum (Fig. 1a). Computed tomography (CT) of the abdomen revealed a large mass located in the stomach with regional lymph node metastasis and direct pancreatic invasion, but there was no evidence of liver metastasis (Fig. 1b). Biopsy specimens of the tumor showed small cells with atypical nuclei and scant cytoplasm (Fig. 2a). Immunohistochemical analysis revealed that the tumor cells were positive for chromogranin A (Fig. 2b) but negative for leukocyte common antigen (Fig. 2c). Based on these findings, we diagnosed primary GSCC with direct pancreatic invasion and multiple lymph node metastases. The clinical stage according to the tumor, node, and metastasis (TNM) classification of malignant tumors was T4bN3aM0 Stage IIIc ${ }^{5}$. Complete removal of the tumor by surgical resection appeared to be difficult because of presence of other organs and multiple lymph node metastasis at the site. After obtaining informed 

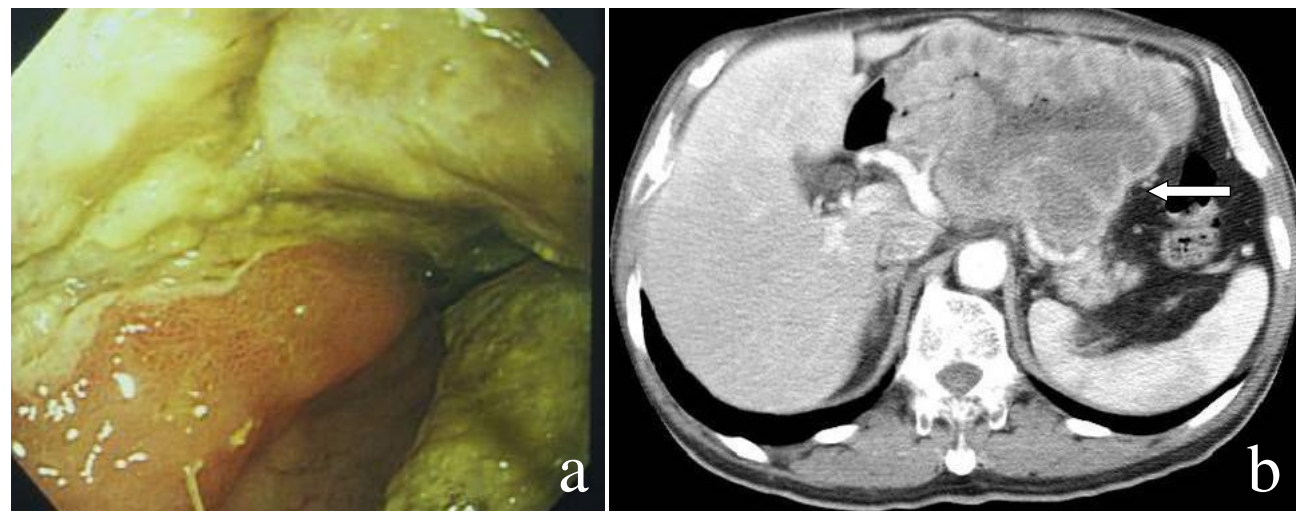

Fig. 1 (a) Esophagogastroduodenoscopic (EGD) image showing a large ulcerated tumor located in the posterior wall of the antrum. (b) Computed tomography (CT) image of the abdomen revealing a large mass involving the stomach with regional lymph node metastasis and direct pancreatic invasion (arrow).

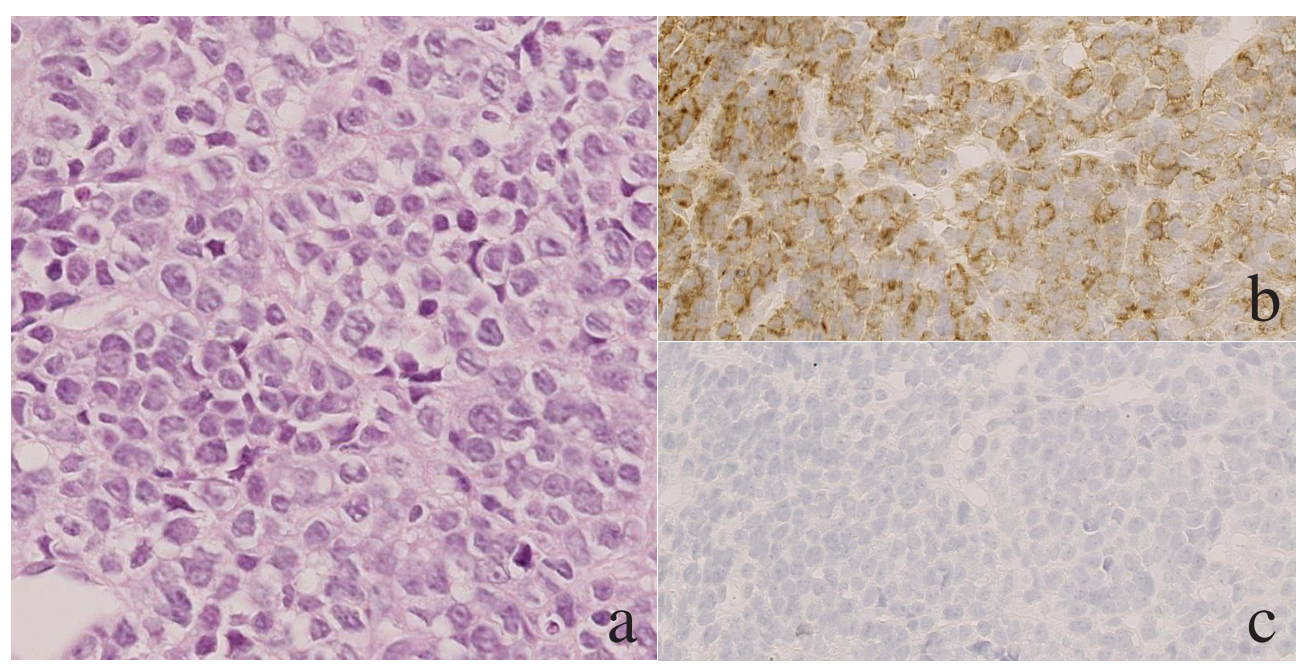

Fig. 2 Pathological findings of the gastric tumor $(\times 400)$. (a) Hematoxylin and eosin staining. Biopsies of the gastric tumor revealed a solid growth of small cell with atypical nuclei and scanty cytoplasm. (b) Immunohistochemical staining for chromogranin A (CGA). The tumor cells were positive for CGA. (c) Immunohistochemical staining for leukocyte common antigen (LCA). The tumor cells were negative for LCA.

consent from the patient, combination chemotherapy was initiated. Irinotecan $70 \mathrm{mg} / \mathrm{m}^{2}$ was administered on days 1 and 15 , and cisplatin $60 \mathrm{mg} / \mathrm{m}^{2}$ was administered intravenously on day 1 , according to the protocol for primary small cell lung carcinoma ${ }^{4}$. Following 2 cycles of chemotherapy, the tumor showed a partial response that was confirmed by EGD (Fig. 3a). Furthermore, the patient's NSE level fell to within the normal range. Following 4 cycles of chemotherapy, he showed a complete remission that was confirmed by EGD, biopsy, and abdominal CT (Fig. 3b, c). He was subsequently diagnosed as showing a complete response. Adverse events that were observed throughout all courses of the treatment included Grade 2 neutropenia, Grade 2 alopecia, Grade 3 nausea, vomiting, appetite loss, and Grade 1 liver dysfunction. Following 9 cycles of IP, the treatment was stopped as per the patient's request. There was no evidence of recurrence during the follow-up period (at 10, 24, 36, and
72 mo post-chemotherapy) (Fig. 4a, b, c, d; Fig. 5a, b, c, d). An abdominal aortic aneurysm was treated with an aortic graft $6 \mathrm{yr}$ after the initial presentation. During the operation, no evidence of recurrence was observed in the abdomen. The patient survived for more than $8 \mathrm{yr}$ since the first diagnosis of cancer.

\section{Discussion}

Reports of extrapulmonary sites of small cell carcinoma are rare and account for $4 \%$ of all the cases ${ }^{6}$. GSCC is recognized as an extremely rare tumor ${ }^{1)}$. Matsusaka et al. first described GSCC in 1976, wherein only 2 cases were reported in a review of about 2000 gastric carcino$\operatorname{mas}^{1)}$.

Diagnosis of GSCC is difficult as it easily infiltrates the submucosa and often coexists with other histological types $^{2)}$. In our case, a definitive diagnosis of GSCC could 


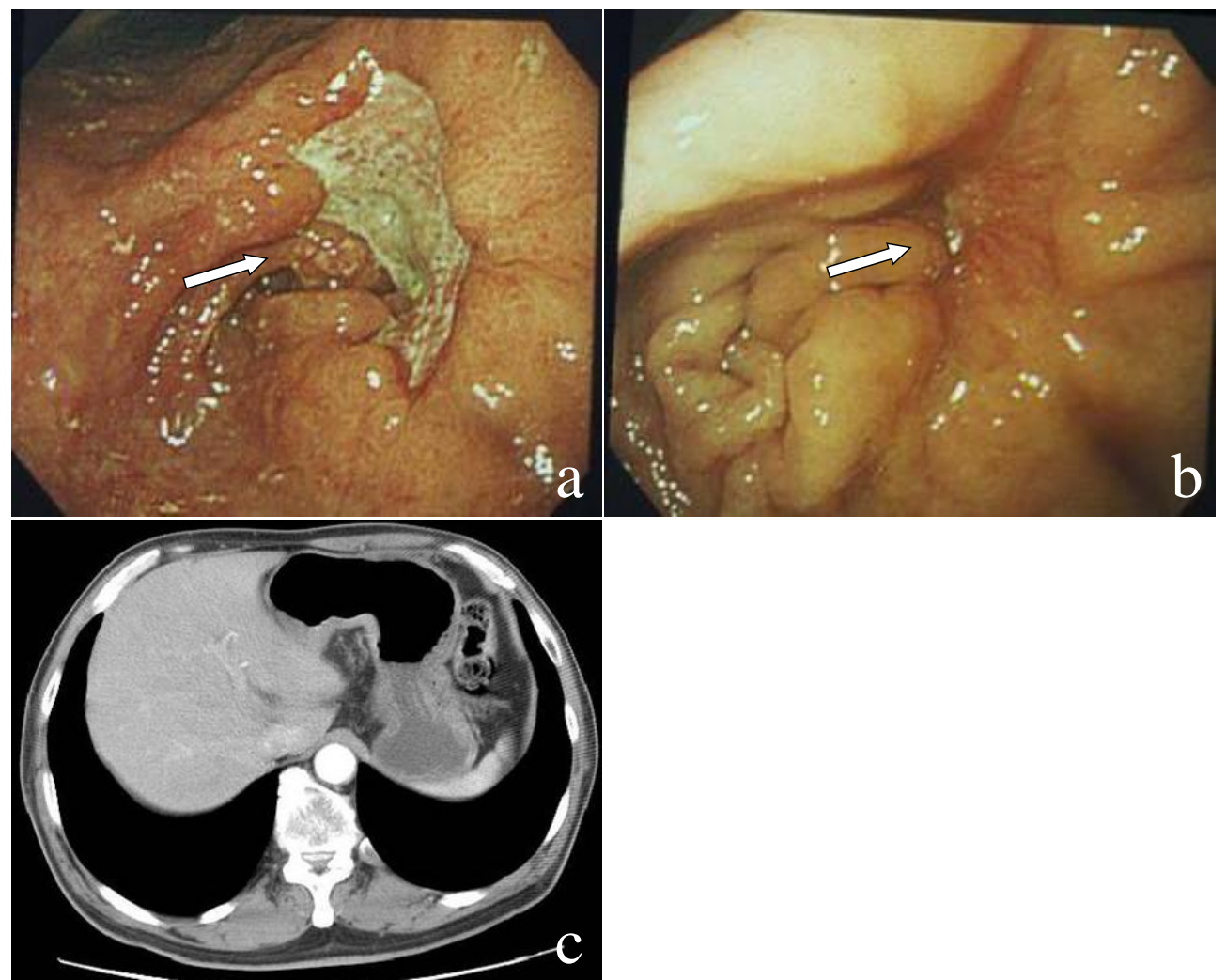

Fig. 3 (a) EGD after 2 cycles of chemotherapy showing a partial response (arrow). (b) EGD after 4 cycles of chemotherapy showing a complete response (arrow). Biopsy revealed no tumor cells. (c) CT after 4 cycles of chemotherapy showing no evidence of tumor.
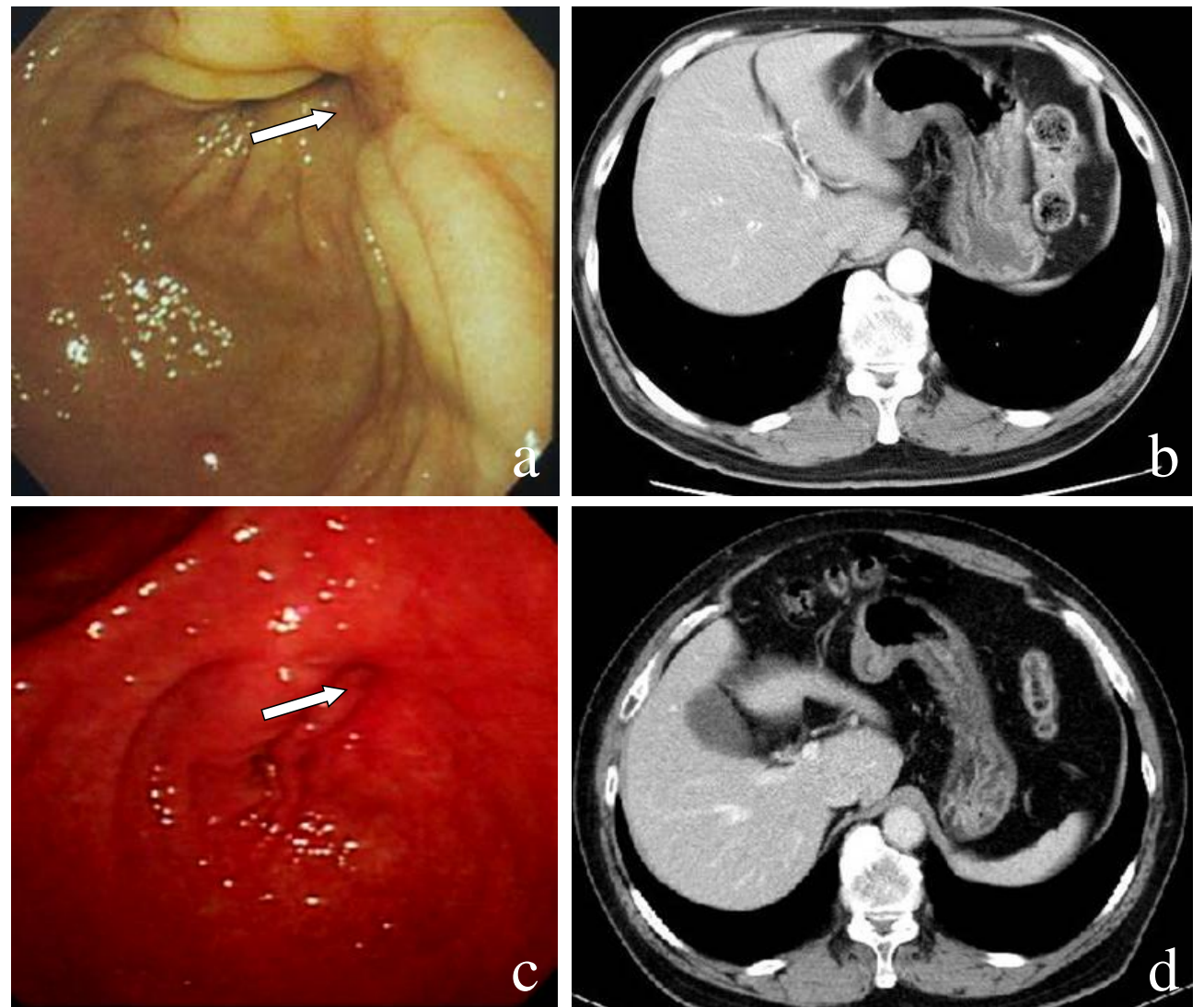

Fig. 4 There was no evidence of recurrence 10 and 24 months (mo) following chemotherapy. (a) EGD image after 10 mo (arrow). (b) CT image after 10 mo. (c) EGD image after 24 months (arrow). (d) CT image after 24 months. 

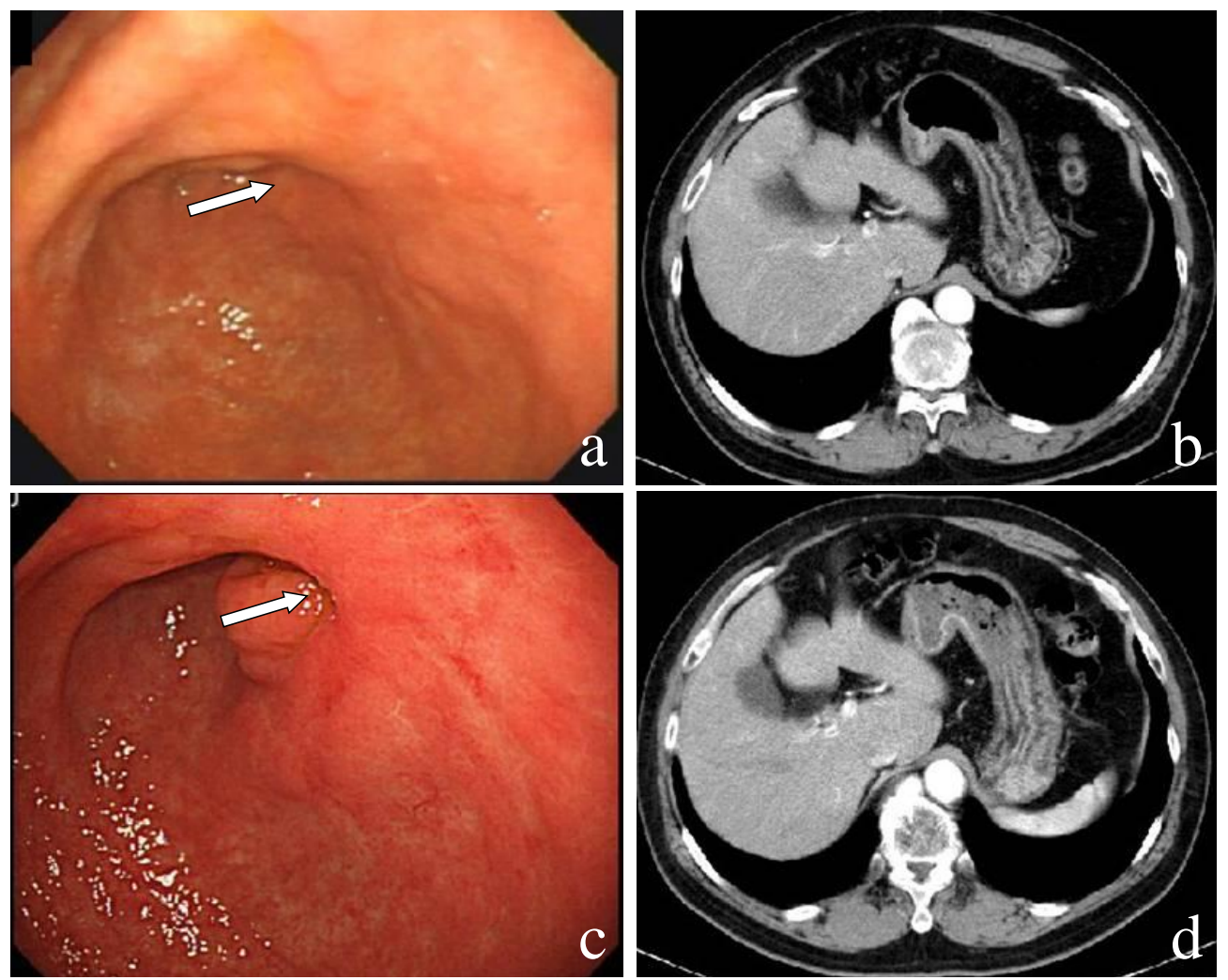

Fig. 5 There was no evidence of recurrence 36 and 72 mo following chemotherapy. (a) EGD image after 36 months (arrow). (b) CT image after 36 months. (c) EGD image after 72 months (arrow). (d) CT image after 72 months.

be made from the biopsy specimens that showed typical histological and immunohistochemical findings.

While few cases of long-term survival have been reported following surgical resection ${ }^{1)}$, the biological manifestations of GSCC is recognized as malignant and aggressive. The outcome is extremely poor due to metastasis or frequent cases of recurrence immediately after surgery, even in cases of the lesion that is limited to the submucosa $^{2,7,8)}$. Namikawa et al. reviewed 107 cases of GSCC and found that $42.1 \%$ patients died within 1 year of recurrence ${ }^{2)}$. In a series of 17 cases reported by Matusi et al. ${ }^{7)}$, the median survival was 9 months. Even in patients without metastatic disease, surgery alone may not be sufficient treatment for cure.

Recently, few studies have reported favorable results with a combined treatment modality of surgery followed by chemotherapy in resectable cases of GSCC, which are similar to that for conventional gastric cancer ${ }^{2,9,10,11)}$. On the other hand, in unresectable cases, chemotherapy showed efficacy for $\mathrm{GSCC}^{3,12,13,14,15)}$. Among the various regimens of chemotherapy reported, cisplatin has been frequently mentioned in the literature ${ }^{2,3,9,10,11,12,14,15,16)}$. It remains unclear whether chemotherapy should be given before or after surgery; however, it may be essential in the treatment of GSCC. We decided to use IP treatment since it is standard care for patients with small cell carcinoma of the lung ${ }^{4}$.
Although the molecular behavior is uncertain, the biological and clinical characteristics of GSCC are similar to those of small cell carcinoma of the lung ${ }^{17,18)}$. Therefore, chemotherapy regimens specific for pulmonary small cell carcinoma may be more effective than conventional chemotherapy for gastric adenocarcinoma. Recently, there have been reports of favorable responses to chemotherapy in extrapulmonary small cell carcinomas. Van Der Gaast A et al. ${ }^{3)}$ reported that 11 patients with extrapulmonary undifferentiated small cell carcinoma who were treated with chemotherapy showed a response rate of $82 \%$. Moreover, IP is widely used not only as a standard treatment for small cell lung cancer, but also for advanced or recurrent gastric adenocarcinoma based on evidence from a randomized controlled trial ${ }^{19)}$. Therefore, IP may be used for cases where pathological diagnosis is difficult in distinguishing between gastric adenocarcinoma and GSCC.

In conclusion, we present a case wherein IP alone was able to cure advanced small cell carcinoma of the stomach. IP may provide survival benefit for patients with GSCC. Considering the high frequency of metastasis of the disease at the time of diagnosis and early systemic relapse after local treatment, IP treatment should be actively considered following diagnosis of small cell carcinoma of the stomach. 


\section{Acknowledgement}

This work was supported, in part, by a Non-Profit Organization Epidemiological \& Clinical Research Information Network (ECRIN).

\section{References}

1) Matsusaka T, Watanabe H and Enjoji M. (1976) Oat-cell carcinoma of the stomach. Fukuoka Igaku Zasshi. 67: 65-73. (in Japanese)

2) Namikawa $T$, Kobayashi M, Okabayashi T, Ozaki $S$, Nakamura $S$, Yamashita K, Ueta H, Miyazaki J, Tamura S, Ohtsuki Y and Araki K. (2005) Primary gastric small cell carcinoma: report of a case and review of the literature. Med Mol Morphol. 38: 256-261.

3) Van Der Gaast A, Verwey J, Prins E and Splinter, TA. (1990) Chemotherapy as treatment of choice in extrapulmonary undifferentiated small cell carcinomas. Cancer. 65: 422-424.

4) Noda K, Nishiwaki $Y$, Kawahara M, Negoro S, Sugiura $T$, Yokoyama A, Fukuoka M, Mori K, Watanabe K, Tamura T, Yamamoto S and Saijo, N. (2002) Irinotecan plus cisplatin compared with etoposide plus cisplatin for extensive small-cell lung cancer. N Engl J Med. 346: 85-91.

5) Sobin L, Gospodarowicz M and Wittekind C (Eds). (2009) TNM Classification of Malignant Tumours, 7th ed. New York: Wiley.

6) Levenson RM, Ihde DC, Matthews MJ, Cohen MH, Gazdar AF, Bunn PA Jr and Minna JD. (1981) Small cell carcinoma presenting as an extrapulmonary neoplasm: Sites of origin and response to chemotherapy. J Natl Cancer Inst. 67: 607-612.

7) Matsui K, Kitagawa M, Miwa A, Kuroda Y and Tsuji M. (1991) Small cell carcinoma of the stomach: a clinicopathologic study of 17 cases. Am J Gastroenterol. 86: 1167-1175.

8) Arai K and Matsuda M. (1998) Gastric small cell carcinoma in Japan. A case report and review of the literature. Am J Clin Oncol. 21: 458-461.

9) Koide N, Suzuki A, Saito H, Sato T, Murakami M, Ota H and Miyagawa S. (2007) Gastric small cell carcinoma successfully treated by surgery and postoperative chemotherapy consisting of cisplatin and S-1: report of a case. Surg Today. 37: 989-994.

10) Hamano R, Hirano T, Tokuoka M, Masuzawa T, Shibata $K$ and Kobayashi T. (2007) A case report of gastric small cell carcinoma with long survival time by adjuvant chemotherapy-reports of chemotherapy regimens for gastric small cell carcinoma. Jpn J Cancer Chemother. 34: 609-613. (In Japanese with English abstract)

11) Sunose Y, Takeyoshi I, Kawate S, Hamada K, Tsutsumi H, Arakawa K, Tohgo N, Ohwada S, Kashiwabara K and Morishita Y.
(2006) A recurrent endocrine cell cancer of the stomach showing almost complete remission after chemotherapy for 1 year. Jpn J Cancer Chemother. 33: 2073-2076. (In Japanese with English abstract)

12) Hosokawa A, Shimada Y, Shirao K, Matsumura Y, Yamada Y, Muro K, Hamaguchi T, Gotoh M and Shimada T. (2004) Long-term survivor of gastric small cell carcinoma. Hepatogastroenterology, 51: 1567-1570.

13) Nakamura $Y$, Otani $S$, Otaka $M$, Shimada $T$, Takahashi $S$, Saito M, Takahashi T, Komatsu M, Suzuki T, Okubo S, Hayashi M and Sasano H. (2005) Gastric small cell carcinoma with marked response to neoadjuvant chemotherapy. Int J Clin Oncol. 10: 348352.

14) Saeki H, Anegawa G, Masuda T, Ohta R, Honda M, Kai T, Kaneko S, Anai H, Nakamura Y and Yokoyama S. (2006) A case of inoperable gastric small cell carcinoma effectively treated by chemotherapy and radiotherapy. Jpn J Cancer Chemother. 33: 977-979. (In Japanese with English abstract)

15) Shimada M, Iwase H, Iyo T, Nakarai K, Kaida S, Indo T, Doi R, Okeya $\mathrm{M}$ and Goto H. (2004) A case of complete response in a primary lesion treated by combined chemotherapy of TS-1 and CDDP for small cell carcinoma of the stomach with liver metastasis. Jpn J Cancer Chemother. 31: 593-596. (In Japanese with English abstract)

16) Kato T, Sato K, Tamahashi N, Yano H and Masuoka H. (2005) A case of small cell carcinoma of the stomach. Jpn J Cancer Chemother. 32: 1473-1475. (In Japanese with English abstract)

17) Hussein AM, Otrakji CL and Hussein BT. (1990) Small cell carcinoma of the stomach. Case report and review of the literature. Dig Dis Sci. 35: 513-518.

18) Ibrahim NBN, Briggs JC and Corbishley CM. (1984) Etrapulmonary oat cell carcinoma. Cancer 1984. 54: 1645-1661.

19) Boku N, Yamamoto S, Fukuda H, Shirao K, Doi T, Sawaki A, Koizumi W, Saito H, Yamaguchi K, Takiuchi H, Nasu J and Ohtsu A, Gastrointestinal Oncology Study Group of the Japan Clinical Oncology Group. (2009) Fluorouracil versus combination of irinotecan plus cisplatin versus S-1 in metastatic gastric cancer: a randomised phase 3 study. Lancet Oncol. 10: 1063-1069. 